\title{
ASYMPTOTIC BEHAVIOR OF SOLUTIONS OF ABSTRACT WAVE EQUATIONS
}

\author{
MARVIN SHINBROT ${ }^{1}$
}

Let $H$ be a complex Hilbert space, and let $A$ be a selfadjoint operator on $H$ satisfying

$$
(A u, u) \geqq 0 \quad \text { for } u \in D(A) .
$$

The abstract wave equations of the title include any equation of the form

$$
d^{2} u / d t^{2}+A u=0 .
$$

A function $t \rightarrow u(t)$ with values in $H$ will be called a solution of (2) if it has two strong derivatives with respect to $t$ and satisfies (2).

Denote differentiation with respect to $t$ by a dot. It is not hard to see that for any solution of (2), the following two quantities are independent of $t$ :

$$
\Pi=\|\dot{u}\|^{2}+\left\|A^{1 / 2} u\right\|^{2}, \quad \Gamma=\|u\|^{2}+\left\|A^{-1 / 2} \dot{u}\right\|^{2} .
$$

When $H=L^{2}\left(R^{n}\right)$ and $A=m^{2} I-\nabla^{2}$ (defined on all functions in $L^{2}\left(R^{n}\right)$ with two distributional derivatives in $\left.L^{2}\left(R^{n}\right)\right)$, Brodsky [1] proved that as $|t| \rightarrow \infty$,

$$
\begin{aligned}
\|u(t)\|^{2} & \rightarrow \Gamma / 2 \\
\left\|A^{1 / 2} u(t)\right\|^{2} & \rightarrow \Pi / 2
\end{aligned}
$$

In this paper, we shall derive two closely related conditions, one necessary and the other sufficient, for every solution of (2) to satisfy (3) and (4). We can then derive Brodsky's result as a corollary.

What we shall prove precisely is the

THEOREM. Let $A$ be a selfadjoint operator satisfying (1). Let ${ }^{2} u_{0} \in$ $D(A), \dot{u}_{0} \in D\left(A^{1 / 2}\right)$. Then, the initial value problem

$$
\begin{aligned}
i i+A u & =0, \\
u(0) & =u_{0}, \quad \dot{u}(0)=\dot{u}_{0},
\end{aligned}
$$

has a unique solution. This solution satisfies

Received by the editors July 21, 1967.

1 Supported, in part, by the National Science Foundation (GP-6632).

${ }^{2}$ At the cost of working with weak solutions, one can weaken the hypotheses on the initial data. 


$$
\|\dot{u}(t)\|^{2}+\left\|A^{1 / 2} u(t)\right\|^{2}=\left\|\dot{u}_{0}\right\|^{2}+\left\|A^{1 / 2} u_{0}\right\|^{2} .
$$

If $A$ has an inverse, and if $\dot{u}_{0} \in D\left(A^{-1 / 2}\right)$, then

$$
\|u(t)\|^{2}+\left\|A^{-1 / 2} \dot{u}(t)\right\|^{2}=\left\|u_{0}\right\|^{2}+\left\|A^{-1 / 2} \dot{u}_{0}\right\|^{2} .
$$

Let $\left\{E_{\lambda}\right\}$ be the spectral family associated with $A$. In order for (3) and (4) to hold for all $u_{0} \in D(A)$ and all $\dot{u}_{0} \in D\left(A^{1 / 2}\right)$, it is necessary that $\left\{E_{\lambda}\right\}$ be continuous; it is sufficient that $\left\{E_{\lambda}\right\}$ be absolutely continuous.

Proof. The uniqueness of solutions of (2) is well known. It can be obtained by taking the scalar product of (2) with $u$ and using (1). For the existence, let $\left\{E_{\lambda}\right\}$ be the spectral family associated with $A$ and define

$$
u(t)=\int_{0}^{\infty} \cos (t \sqrt{ } \lambda) d E_{\lambda} u_{0}+\int_{0}^{\infty} \frac{\sin (t \sqrt{ } \lambda)}{\sqrt{ } \lambda} d E_{\lambda} \dot{u}_{0} .
$$

A direct computation shows that $u(t)$ satisfies (2) and (5).

To prove (7), we notice that

$$
\begin{aligned}
\|u(t)\|^{2}=\int_{0}^{\infty} & {\left[\cos ^{2}(t \sqrt{ } \lambda) d\left\|E_{\lambda} u_{0}\right\|^{2}\right.} \\
& +\frac{2}{\sqrt{ } \lambda} \sin (t \sqrt{ } \lambda) \cos (t \sqrt{ } \lambda) \operatorname{Re}\left\{d\left(u_{0}, E_{\lambda} \dot{u}_{0}\right)\right\} \\
& \left.+\frac{1}{\lambda} \sin ^{2}(t \sqrt{ } \lambda) d\left\|E_{\lambda} \dot{u}_{0}\right\|^{2}\right],
\end{aligned}
$$

while

$$
\begin{aligned}
\left\|A^{-1 / 2} \dot{u}(t)\right\|^{2}=\int_{0}^{\infty} & {\left[\sin ^{2}(t \sqrt{ } \lambda) d\left\|E_{\lambda} u_{0}\right\|^{2}\right.} \\
& \quad-\frac{2}{\sqrt{ } \lambda} \sin (t \sqrt{ } \lambda) \cos (t \sqrt{ } \lambda) \operatorname{Re}\left\{d\left(u_{0}, E_{\lambda} \dot{u}_{0}\right)\right\} \\
& \left.+\frac{1}{\lambda} \cos ^{2}(t \sqrt{ } \lambda) d\left\|E_{\lambda} \dot{u}_{0}\right\|^{2}\right] .
\end{aligned}
$$

Therefore,

$$
\begin{aligned}
\|u(t)\|^{2}+\left\|A^{-1 / 2} \dot{u}(t)\right\|^{2} & =\int_{0}^{\infty}\left[d\left\|E_{\lambda} u_{0}\right\|^{2}+\frac{1}{\lambda} d\left\|E_{\lambda} \dot{u}_{0}\right\|^{2}\right] \\
& =\left\|u_{0}\right\|^{2}+\left\|A^{-1 / 2} \dot{u}_{0}\right\|^{2} .
\end{aligned}
$$

(6) can be proved in the same way. 
To prove the necessity of the continuity of $\left\{E_{\lambda}\right\}$, suppose $\left\{E_{\lambda}\right\}$ were not continuous. Then, $A$ would have an eigenvalue $\lambda_{0}$. Take $u_{0}$ to be an eigenfunction with this eigenvalue, and take $\dot{u}_{0}$ to be zero. Because of (1), $\lambda_{0}$ is nonnegative. Thus, we can write

$$
u(t)=u_{0} \cos \left(t \sqrt{ } \lambda_{0}\right),
$$

and it is immediate that this is the solution of (2) and (5). On the other hand,

$$
\|u(t)\|^{2}=\left\|u_{0}\right\|^{2} \cos ^{2}\left(t \sqrt{ } \lambda_{0}\right),
$$

and this has no limit as $|t| \rightarrow \infty$, unless $\lambda_{0}=0$.

If $\lambda_{0}=0$, however, we take $u_{0}$ to be zero and $\dot{u}_{0}$ to be an eigenfunction with eigenvalue zero. In this case, the solution of (2) and (5) is $u(t)=\dot{u}_{0} t$, and again $\|u(t)\|^{2}=\left\|\dot{u}_{0}\right\|^{2} t^{2}$ has no limit as $|t| \rightarrow \infty$. This proves that the continuity is necessary.

To prove the last clause in the theorem, we return to (8). We have

$$
\begin{aligned}
\|u(t)\|^{2}=\frac{1}{2} \int_{0}^{\infty}\left[d\left\|E_{\lambda} u_{0}\right\|^{2}+\frac{1}{\lambda} d\left\|E_{\lambda} \dot{u}_{0}\right\|^{2}\right] & \\
+\frac{1}{2} \int_{0}^{\infty} & {\left[\cos (2 t \sqrt{ } \lambda) d\left\|E_{\lambda} u_{0}\right\|^{2}\right.} \\
& +\frac{2}{\sqrt{ } \lambda} \sin (2 t \sqrt{ } \lambda) \operatorname{Re}\left\{d\left(u_{0}, E_{\lambda} \dot{u}_{0}\right)\right\} \\
& \left.-\frac{1}{\lambda} \cos (2 t \sqrt{ } \lambda) d\left\|E_{\lambda} \dot{u}_{0}\right\|^{2}\right] .
\end{aligned}
$$

By hypothesis, $\left\|E_{\lambda} u_{0}\right\|^{2},\left(u_{0}, E_{\lambda} \dot{u}_{0}\right)$, and $\left\|E_{\lambda} \dot{u}_{0}\right\|^{2}$ are absolutely continuous. Thus, the Riemann-Lebesgue lemma shows that

$$
\begin{aligned}
\|u(t)\|^{2} & \rightarrow \frac{1}{2} \int_{0}^{\infty}\left[d\left\|E_{\lambda} u_{0}\right\|^{2}+\frac{1}{\lambda} d\left\|E_{\lambda} \dot{u}_{0}\right\|^{2}\right] \\
& =\frac{1}{2}\left[\left\|u_{0}\right\|^{2}+\left\|A^{-1 / 2} \dot{u}_{0}\right\|^{2}\right] .
\end{aligned}
$$

This is (3). (4) can be proved similarly.

REMARK. The proof of the theorem shows that for (3) and (4) to hold for a particular $u_{0}$ and $\dot{u}_{0}$, it is not really necessary for the spectral family $\left\{E_{\lambda}\right\}$ to be absolutely continuous. Indeed, if it is merely true that $u_{0}$ and $\dot{u}_{0}$ lie in the absolutely continuous subspace [2, p. 436] of $A$, so that $\left\|E_{\lambda} u_{0}\right\|^{2},\left(u_{0}, E_{\lambda} \dot{u}_{0}\right)$, and $\left\|E_{\lambda} \dot{u}_{0}\right\|^{2}$ are absolutely 
continuous, the sufficiency part of the theorem goes through just as well.

Now, let $H=L^{2}\left(R^{n}\right)$. Let $D\left(A_{0}\right)$ be the set of all functions in $L^{2}\left(R^{n}\right)$ having two distributional derivatives in $L^{2}\left(R^{n}\right)$. On $D\left(A_{0}\right)$, define

$$
A_{0} u=m^{2} u-\nabla^{2} u,
$$

where $m \geqq 0$. It is known [2, p. 444] that the absolutely continuous subspace of $A_{0}$ is all of $L^{2}\left(R^{n}\right)$. The proof depends on the fact that $A_{0}$ is unitarily equivalent (via the Fourier transform) to multiplication by $m^{2}+|x|^{2}$. Therefore, we have the

Corollary (Brodsky). Let $A_{0}$ be defined as above, and let $u_{0} \in D\left(A_{0}\right)$, $\dot{u}_{0} \in D\left(A_{0}^{1 / 2}\right)$ (see footnote 2 ). Let $u$ be the solution of the abstract wave equation associated with $A_{0}$. Then,

$$
\lim \|u(t)\|^{2}=\Gamma / 2, \quad \lim \left\|A_{0}^{1 / 2} u(t)\right\|^{2}=\Pi / 2 .
$$

1. A. R. Brodsky, On the asymptotic behavior of solutions of the wave equations, Proc. Amer. Math. Soc. 18 (1967), 207-208.

2. S. T. Kuroda, On the existence and the unitary property of the scattering operator, Nuovo Cimento 12 (1959), 431-454.

NORTHWESTERN UNIVERSITY 